\title{
Le numérique en éducation : apprendre en ouvrant les murs de la classe
}

Alain Stockless

Université du Québec à Montréal

L'idée d'ouvrir les murs de la classe peut prendre différentes formes, et ce, autant sur le plan de la redéfinition des espaces que dans les pratiques pédagogiques. II va de soi que l'on assiste à une ouverture identitaire de l'altérité relativement au numérique en éducation. II y a peu de doute sur le fait que le numérique permet aux apprenants et aux enseignants d'explorer et de faire vivre de nouvelles expériences éducatives qui autrement ne pourraient pas être possible. Ainsi, c'est dans le sens d'innover, d'explorer et de partager que cette thématique prend son sens. C'est également s'ouvrir vers d'autres perspectives pour [mieux] voir ce qui se passe à l'extérieur et c'est aussi autant d'occasions pour faire entrer un monde à découvrir dans sa classe. Déjà dans les années 1930, Dewey (1938) parlait d'expérience éducative hors de la classe. Pour Erstad, de nouveaux espaces d'apprentissage, par exemple le Web, les bibliothèques, les musées, permettent une perméabilité des frontières entre l'école et la vie de tous les jours. C'est ce qu'il appelle " the expanded classroom » (Erstad, 2013; Erstad, Gilje, Sefton-Green et Arnseth, 2016). Dans cette perspective, certains parlent même de Pedagocization of Everyday Life (Bernstein, 2001; Sefton-Green, 2016).

Évidemment, la notion de murs est symbolique, mais sa représentation reste lourde de sens et elle est associée à une structure solidement ancrée dans un écosystème que nous avons longtemps considéré comme étant le lieu où l'on pouvait formellement apprendre. Le développement technologique des années 1990 avec le Web a créé une fissure dans les murs de la classe. Cette brèche rend bien compte d'un changement de posture quant à la possession du savoir et des interactions entre les différents utilisateurs. Des innovations pédagogiques sont maintenant possibles. D'autres auteurs parlent plutôt de disruptive technologies (Conole, de Laat, Dillon et Darby, 2008): les espaces d'apprentissage se décloisonnent et nous n'avons qu'à penser au concept de classe inversée, aux cours hybrides où le rapport espace-temps prend un tout autre sens. Aussi, ces modalités sont l'occasion d'apprendre au sein d'une communauté où les interactions permettent aux apprenants de vivre des expériences éducatives en ligne riches en ce qui a trait au partage de savoirs (Garrison, 2017). Décloisonner la classe c'est également repenser physiquement la classe et nous pensons ici aux travaux du Future Classroom Lab (http://fcl.eun.org/) et au redesign des espaces d'apprentissage pour rendre les apprenants plus actifs (Adams Becker et al., 2017). 


\section{Du processus d'intégration des TIC vers une approche plus globale}

Apprendre avec le numérique peut engendrer de nouveaux types d'interactions et d'apprentissage, même s'il est évidemment possible de reproduire des pratiques pédagogiques existantes sans faire ressortir la plus value de ces outils. Dès les années 1990, les travaux de Sandholtz, Ringstaff et Dwyer (1997) et par la suite ceux de Cuban, Kirkpatrick et Peck (2001) ont montré qu'il n'est pas suffisant de rendre des outils technologiques disponibles pour voir des changements dans les usages pédagogiques autres que celles de reproduire des pratiques existantes. Aujourd'hui, nous retenons bien ces leçons, mais nous constatons souvent que pour soutenir l'apprentissage, une réflexion sur la façon dont on intègre le numérique reste toujours pertinente. Une utilisation optimale du numérique semble en apparence simple. Selon le rapport New Horizon de 2015, nous avons toutes les connaissances scientifiques nécessaires pour résoudre à court terme les problématiques relatives à l'intégration du numérique en classe notamment en ce qui a trait aux modèles en formation initiale et pour le développement professionnel (Johnson, Adams Becker, Estrada et Freeman, 2015). Nous n'avons qu'à penser au modèle SAMR (substitution, augmentation, modification et redéfinition) de Puentedura (2010) ou Romero (2015) sur le passage de la consommation de contenu vers des usages de cocréation participative. Sinon, le TPACK (Technology, Pedagogy, and Content Knowledge) de Koehler et Mishra (2008) propose une perspective plutôt systémique dans laquelle l'enseignant doit maitriser des dimensions relatives à des connaissances sur le contenu, la pédagogie et les technologies. Ces modèles, nous amènent à réfléchir et à renouveler des pratiques pédagogiques et surtout à la façon dont le numérique est utilisé en contexte éducatif. Alors, autant aujourd'hui que dans les années 1990, les différents intervenants en éducation ont pressenti des usages du numérique sous une perspective de changement. Un des problèmes réside justement dans l'idée que trop souvent, l'outil technologique, en tant qu'objet, est nécessairement un vecteur d'innovation engendrant un changement pédagogique. Les travaux antérieurs sur les usages pédagogiques du numérique évoqués plus tôt ont fait ressortir un apport limité au soutien à l'apprentissage qui risque bien de se reproduire. Or, le terme « intégration » du numérique signifie que l'on ajoute un outil numérique à un processus [pédagogique] déjà existant. Cette démarche peut difficilement mener à une utilisation optimale du numérique pour soutenir les apprentissages puisqu'il est vu comme un objet pour améliorer une situation existante plutôt qu'à amorcer une réflexion sur l' intention pédagogique. Une réflexion en amont sur ce que l'on veut faire et la manière dont on veut enseigner est indispensable pour définir les choix pédagogiques et les ressources appropriées. Ouvrir les murs de la classe avec le numérique devrait être vu comme une opportunité pour se questionner sur la place du numérique dans un contexte éducatif, mais aussi pour réfléchir sur ses apports au processus d'apprentissage.

C'est précisément sur cette thématique que s'est déroulé en 2017 le colloque de la Communauté pour I'Innovation et la Recherche sur les Technologies dans l'enseignement et l'Apprentissage (CIRTA). Ce premier numéro de médiations et médiatisations présente des articles complémentaires aux actes de colloque (Stockless, Lepage et Plante, 2018) sur le numérique en éducation et l'idée d'ouvrir les murs de la classe sur le monde. Tout d'abord, Carole Calistri et Virginie Lapique ont cosigné un article qui a pour objet une expérimentation de classe inversée dans le cadre de la formation des enseignants. Matthieu Petit nous présente un article sur la supervision de stage à distance à l'aide du numérique. Pour Caterina Ciampi, Keven Doyon-Lacasse, Sabrina Priego, Min-Hsun Chiang et Meei-Ling Liaw, l'idée d'ouvrir les murs de la classe s'est réalisée dans le cadre d'une recherche sur la co-construction des connaissances des enseignants en formation ou en service à travers un projet interculturel télécollaboratif. Anastassis Kozanitis et Claude Quevillon Lacasse nous présentent une recherche exploratoire sur l'utilisation des TICE pour soutenir les pédagogies actives en contexte d'enseignement universitaire. Ensuite, François Lewis et Patrick Plante présentent une recherche sur la création et 
l'évaluation d'un jeu sérieux. Pour ouvrir les murs de la classe, le texte de Mathieu Thibault et Fabienne Venant porte sur le potentiel de Twitter pour apprendre en mathématique. Quant à la rubrique débat et discussion, Mathieu Cisel a signé l'article Certificats de MOOC : débats passés et présents sur un objet en évolution rapide. Ce premier numéro est également l'occasion de s'interroger sur le champ de la revue. Ainsi, Cathia Papi nous présente un entretien avec Daniel Peraya permettant de revenir sur l'émergence et le sens donné aux concepts de médiation et médiatisation. Alain Stockless propose en clôture du numéro un entretien avec Brigitte Albero sur les enjeux actuels de la recherche sur le numérique.

Bonne lecture !

\section{Bibliographie}

ADAMS BECKER, S., CUMMINS, M., DAVIS, A., FREEMAN, A., HALL GIESINGER, C. ET ANANTHANARAYANAN, V. (2017). NMC Horizon Report: 2017 Higher Education Edition. Austin: The New Media Consortium.

BERNSTEIN, B. (2001). From pedagogies to knowledges. Dans A. Morais, I. Neves, B. Davies \& H. Daniels (dir.), Towards a sociology of pedagogy: the contribution of Basil Bernstein to research (p. 363-368). New York: Peter Lang.

CONOLE, G., de LAAT, M., DILLON, T. et DARBY, J. (2008). 'Disruptive technologies', 'pedagogical innovation': What's new? Findings from an in-depth study of students' use and perception of technology. Computers \& Education, 50(2), 511-524. doi: 10.1016/j.compedu.2007.09.009

CUBAN, L., KIRKPATRICK, H. ET PECK, C. (2001). High Access and Low Use of Technologies in High School Classrooms: Explaining an Apparent Paradox. American Educational Research Journal, 38(4), 813-834.

DEWEY, J. (1938). Experience and education. New York: Free Press.

ERSTAD, O. (2013). Digital learning lives: TRAJECTORIES, literacies, and schooling. : Peter Lang.

ERSTAD, O., GILJE, Ø., SEFTON-GREEN, J. et ARNSETH, H. C. (2016). Learning Identities, Education and Community: Young Lives in the Cosmopolitan City. : Cambridge University Press.

GARRISON, D. R. (2017). E-learning in the 21st century: A framework for research and practice. (3 éd.). New York: Taylor \& Francis.

JOHNSON, L., ADAMS BECKER, S., ESTRADA, V. ET FREEMAN, A. (2015). NMC Horizon Report: 2015 Higher Education Edition. Austin: The New Media Consortium.

KOEHLER, M. J. ET MISHRA, P. (2008). Introducing TPCK. Dans M. J. Koehler \& P. Mishra (dir.), Handbook of Technological Pedagogical Content Knowledge TPCK for Educators (p. 3-29). New York: Routledge.

PUENTEDURA, R. (2010). SAMR and TPCK: Intro to Advanced Practice. Repéré à http://hippasus.com/resources/sweden2010/SAMR_TPCK_IntroToAdvancedPractice.pdf

ROMERO, M. (2015). Usage pédagogique des TIC : de la consommation passive à la cocréation participative Vitrine technologie-éducation. Repéré le 21 juin 20182018 à https://www.vteducation.org/fr/articles/collaboration-avecles-technologies/usages-pedagogiques-des-tic-de-la-consommation-a-la

SANDHOLTZ, J. H., RINGSTAFF, C. ET DWYER, D. C. (1997). La classe branchée: enseigner à l'ère des technologies. Montréal: Chenelière/McGraw-Hill.

SEFTON-GREEN, J. (2016). Can Studying Learning across Contexts Change Educational Research or Will it Lead to the Pedagocization of Everyday Life? (Learning across Contexts in the Knowledge Society (p. 243-251): Springer.

STOCKLESS, A., LEPAGE, I. ET PLANTE, P. (Dir.). (2018). Actes de colloque de la CIRTA 2017. Ouvrir les murs de la classe avec le numérique. Montréal: CIRTA. 